S1 Table: Description of Predictor Variables Included in the Analyses.

Variable

\section{Socioeconomic variables}

Age

Gender

Race

Nativity

Employment

Education

Marital status

Family size

Home ownership

Number of rooms

Food expenditures-at home

Food expenditures-away from home

Ratio of family income to poverty level

Health insurance

BMI

\section{Dental variables}

Routine dental care

Decayed teeth

Periodontal disease

\section{Medical conditions}

Asthma

Arthritis

Diabetes

Stroke

Heart attack

Coronary heart disease

Heart failure

Angina

High cholesterol

Hypertension

Gout
Age in years at screening (18 to $80 \mathrm{y}$ )

Male, Female

Non-Hispanic White, Non-Hispanic Black, Mexican American, Other Hispanic, Other race

US, Other

Yes, No

Less than 9th grade, 9-11th grade, HS grad/GED or equivalent, some college or AA degree, or College grad or above

Married, Widowed, Divorced, Separated, Never married, or Living with partner

Total number of people in the Family (1 to 7)

Owned/bought, Rented, or Other arrangement

1 to 15 rooms

Money spent at supermarket or grocery store

Money spent on eating out, carry out, or delivered food

0 to 5

Covered by health insurance (yes, no)

Body Mass Index

Visited the dentist in the past year for check-up, examination, or cleaning

Number of decayed teeth based on clinical examination.

Having 2 or more sites with clinical attachment loss of greater than $5 \mathrm{~mm}$ and one or more site with probing depth greater than $4 \mathrm{~mm}$.

Ever been told you have asthma (yes, no)

Doctor ever said you had arthritis (yes, no)

Doctor told you have diabetes (yes, no, borderline)

Ever told you had a stroke (yes, no)

Ever told you had heart attack (yes, no)

Ever told you had coronary heart disease (yes, no)

Ever told had congestive heart failure (yes, no)

Ever told you had angina/angina pectoris (yes, no)

Doctor told you - high cholesterol level (yes, no)

Ever told you had high blood pressure (yes, no)

Doctor ever told you that you had gout? (yes, no) 